\title{
QUALIDADE DE KIWI ARMAZENADO EM DUAS TEMPERATURAS SOB ATMOSFERA CONTROLADA E COM ELIMINAÇÃO DE ETILENO
}

\author{
QUALITY OF KIWIFRUIT STORED UNDER TWO TEMPERATURES AND CONTROLLED \\ ATMOSPHERE WITH ETHYLENE ABSORPTION
}

\author{
Sérgio Miguel Mazaro ${ }^{1}$ Auri Brackmann ${ }^{2}$ Lindolfo Storck $^{3}$
}

\section{RESUMO}

Com objetivo de avaliar o efeito das temperaturas de armazenamento, concentrações de $\mathrm{O}_{2}$ e $\mathrm{CO}_{2}$ em câmaras de atmosfera controlada (AC) e absorção e eliminação do etileno no armazenamento refrigerado $(A R)$ e em AC sobre as qualidades físico-químicas e organolépticas de kiwis, foi conduzido um experimento com as cultivares Bruno e Hayward. As temperaturas de armazenamento foram $-0,5^{\circ} \mathrm{C}$ e $0,5^{\circ} \mathrm{C}$ e a umidade relativa do ar de 97\%. As condições de AR avaliadas foram: sem absorção do etileno; com absorção do etileno através de filtros químicos e eliminação de etileno através da ventilação da câmara. Em $A C$, os frutos foram armazenados em atmosfera contendo: $2 \mathrm{kPa}$ de $\mathrm{O}_{2}$ com 5 e $7 \mathrm{kPa}$ de $\mathrm{CO}_{2}$, com ou sem absorção do etileno através de filtros químicos. As avaliações foram realizadas após três meses de armazenamento para os frutos de ambas as cultivares conservadas em AR. Em AC, os frutos foram avaliados após três e oito meses de armazenamento para as cultivares Bruno e Hayward, respectivamente. Os resultados mostraram que o armazenamento a $-0,5^{\circ} \mathrm{C}$ propiciou, nas duas cultivares, melhor manutenção da firmeza de polpa e mais alta acidez titulável que o armazenamento a $0,5^{\circ} \mathrm{C}$, tanto na abertura das câmaras, como após cinco dias a $20^{\circ} \mathrm{C}$. Nas condições de atmosfera controlada com absorção do etileno, através de filtros químicos, os frutos apresentaram melhor qualidade durante o período de armazenamento, em comparação com os frutos sem absorção de etileno. Em AC, os frutos armazenados na condição de $2 \mathrm{kPa}$ de $\mathrm{O}_{2} \mathrm{com}$ $7 \mathrm{kPa}$ de $\mathrm{CO}_{2}$ se mantiveram, de modo geral, com maior firmeza de polpa e acidez titulável do que aqueles armazenados em $2 \mathrm{kPa}$ de $\mathrm{O}_{2}$ com $5 \mathrm{kPa}$ de $\mathrm{CO}_{2}$. A ventilação da câmara no AR, para eliminação do etileno, não foi eficiente no controle da perda de qualidade dos frutos. A cv. Hayward apresentou maior potencial de armazenamento que a cv. Bruno.

Palavras-chave: Actinidia, qualidade pós-colheita, frigoconservação.

\section{SUMMARY}

The experiment was carried out aiming to evaluate the effect of storage temperature and $\mathrm{O}_{2}$ and $\mathrm{CO}_{2}$ concentrations during controlled atmosphere (CA) storage, as well as the effect of ethylene absorption and elimination during cold storage and CA storage on postharvest quality of 'Bruno' and 'Hayward' kiwifruits. The storage temperatures were $-0.5^{\circ} \mathrm{C}$ and $0.5^{\circ} \mathrm{C}$ with $97 \%$ relative humidity. The cold storage conditions evaluated were: without ethylene absorption; with chemical ethylene absorption, and elimination of ethylene with ventilation of storage rooms. The CA conditions evaluated were: $2 \mathrm{kPa}$ of $\mathrm{O}_{2}$ with 5 and $7 \mathrm{kPa}$ of $\mathrm{CO}_{2}$, with or without chemical ethylene absorption. The evaluations were done after three months for the both cultivars in cold storage. In CA storage the evaluations were done after three and eight months storage for 'Bruno' and 'Hayward' respectively. The results show that the temperature of $-0.5^{\circ} \mathrm{C}$ mantained in both cultivars, higher flesh firmness and higher titratable acidity, at chamber opening and after 5 days at $20^{\circ} \mathrm{C}$ than $0.5^{\circ} \mathrm{C} . \mathrm{CA}$ conditions with chemical ethylene absorption mantained higher quality than without ethylene absorption. In CA storage $2 \mathrm{kPa}$ of $\mathrm{O}_{2}$ and $7 \mathrm{kPa}$ of $\mathrm{CO}_{2}$ resulted in fruit with higher flesh firmness and higher titratable acidity than $2 \mathrm{kPa}$ of $\mathrm{O}_{2}$ and $5 \mathrm{kPa}$ of $\mathrm{CO}_{2}$. Chamber ventilation in cold storage for ethylene removal was not efficient to preserve fruit quality Cultivar Hayward showed higher storability than cultivar Bruno.

Key words: Actinidia, postharvest quality, cold storage.

\section{INTRODUÇÃO}

A colheita do kiwi no sul do Brasil ocorre nos meses de abril a maio. $\mathrm{O}$ armazenamento adequado permite que o produto seja ofertado de forma escalonada durante a maior parte do ano (EPAGRI, 1996).

\footnotetext{
${ }^{1}$ Engenheiro Agrônomo, Mestre, Professor da Escola Agrotécnica Federal - Dois Vizinhos - PR.

2 Engenheiro Agrônomo, Doutor, Professor do Departamento de Fitotecnia, Universidade Federal de Santa Maria (UFSM), 97105-900

Santa Maria, RS. Bolsista do CNPq. E-mail: brackmann@ccr.ufsm.br. Autor para correspondência.

${ }^{3}$ Engenheiro Agrônomo, Doutor, Professor do Departamento de Fitotecnia, UFSM, RS. Bolsista do CNPq.
} 
No armazenamento refrigerado convencional (AR), onde são controladas a temperatura e a umidade relativa das câmaras, o alto metabolismo dos frutos conduz a um rápido amadurecimento. Em atmosfera controlada (AC), há uma maior manutenção da qualidade dos frutos, permitindo um prolongamento do período de conservação. $\mathrm{O}$ efeito da atmosfera controlada, contendo baixo $\mathrm{O}_{2}$ e alto $\mathrm{CO}_{2}$, sobre a conservação da qualidade de kiwi tem sido comprovada por diversos autores (HARRIS, 1976; MITCHELL et al., 1982; TONINI et al., 1996; PARMENTIER \& PROFT, 1997). Porém, o aspecto mais relevante da conservação do kiwi é a necessidade do controle do etileno, porque esse hidrocarboneto induz a maturação dos frutos (HARRIS \& REID, 1981; McDONALD \& HARMAN, 1982).

Por se tratar de uma cultura recente, informações sobre o armazenamento de kiwi, produzido nas condições edafo-climáticas brasileiras, são limitadas. Além disso, a cv. Bruno é pouco estudada internacionalmente por sua reduzida importância comercial em outros países. Como existe uma expectativa otimista para a expansão da cultura, faz-se necessário pesquisar as condições de armazenamento que possibilitem a manutenção da qualidade dos frutos durante o período pós-colheita. Os objetivos deste trabalho foram de avaliar o efeito da temperatura de armazenamento, concentrações de $\mathrm{O}_{2}$ e $\mathrm{CO}_{2}$ em câmaras de atmosfera controlada, e a eliminação do etileno do ambiente das câmaras de AR e AC sobre as qualidades físico-químicas e organolépticas das cultivares de kiwi Hayward e Bruno.

\section{MATERIAL E MÉTODOS}

O experimento foi conduzido no Núcleo de Pesquisa em Pós-colheita (NPP) do Departamento de Fitotecnia da Universidade Federal de Santa Maria, RS, no período de abril a dezembro de 1997. Os frutos das cultivares Hayward e Bruno foram colhidos em um pomar comercial da empresa Kiwistrin, localizada em Farroupilha-RS. A colheita foi realizada no dia 20 de abril de 1997, quando os frutos apresentavam em torno de $7,0^{\circ}$ Brix de sólidos solúveis totais (SST). Após a colheita, os frutos foram transportados até o NPP, em Santa Maria, onde então foram selecionados, eliminando aqueles com lesões, defeitos e baixo calibre e homogeneizadas as unidades experimentais de 30 frutos cada. $\mathrm{O}$ delineamento experimental utilizado foi o inteiramente casualizado com três repetições. Os frutos foram acondicionados em caixas plásticas com capacidade para $20 \mathrm{~kg}$, e então armazenados em minicâmaras experimentais de AC com volume de 232 litros, que permaneceram no interior de duas câma- ras frigoríficas de $45 \mathrm{~m}^{3}$, sendo uma com temperatura de $-0,5^{\circ} \mathrm{C}$ e a outra com $+0,5^{\circ} \mathrm{C}$, e umidade relativa do ar em torno de $97 \%$, que foi monitorada semanalmente, através de um psicrômetro e com auxílio de uma tabela psicrométrica.

Foram avaliadas nove condições de armazenamento. As temperaturas de armazenamento foram $-0,5^{\circ} \mathrm{C}$ e $0,5^{\circ} \mathrm{C}$ e a umidade relativa do ar de 97\%. As condições de AR avaliadas foram: sem absorção do etileno; com absorção do etileno através de filtros químicos e eliminação de etileno através de aeração da câmara. Em AC, os frutos foram armazenados em atmosfera contendo: $2 \mathrm{kPa}$ de $\mathrm{O}_{2}$ com 5 e $7 \mathrm{kPa}$ de $\mathrm{CO}_{2}$, com ou sem absorção do etileno através de filtros químicos.

A instalação da atmosfera controlada e seu monitoramento durante o período do experimento, bem como da atmosfera refrigerada, seguiram a metodologia descrita por BRACKMANN et al. (1999).

As concentrações de etileno foram monitoradas com auxílio de um cromatógrafo gasoso, equipado com coluna "Poropak N" e detector de ionização de chama. A temperatura da coluna foi de $90^{\circ} \mathrm{C}$, do injetor de $140^{\circ} \mathrm{C}$ e do detector de $200^{\circ} \mathrm{C}$. A determinação era feita com uma amostra de $1 \mathrm{~m} \ell$ de gás de cada câmara. Para os tratamentos com eliminação do etileno, foi realizada uma constante absorção química desse gás com um filtro contendo um produto absorvente à base de permanganato de potássio. $\mathrm{O}$ ar da câmara foi constantemente circulado pelo filtro, com auxílio de uma bomba de membrana com fluxo de $240 \ell \cdot \mathrm{h}^{-1}$. Mensalmente, foi realizada uma substituição do produto absorvente para evitar a saturação do mesmo e, conseqüentemente, perda do poder de absorção do etileno. No tratamento no qual se efetuou a ventilação da minicâmara, foi injetado ar atmosférico livre de etileno, durante 30 minutos, uma vez por dia, utilizando uma bomba de membrana com fluxo de $240 \ell \cdot \mathrm{h}^{-1}$. Esse procedimento renova o ar da minicâmara e evita o acúmulo de etileno.

As avaliações físico-químicas dos parâmetros de qualidade dos frutos foram realizadas após três e oito meses de armazenamento. Entretanto, para a cultivar Bruno, em função de seu avançado grau de amadurecimento, as análises foram realizadas somente aos três meses de armazenamento. Para a cv. Hayward, as avaliações foram aos três meses e também aos oito meses de armazenamento, mas somente com as condições de $\mathrm{AC}$, devido ao fato que, em AR, os frutos já apresentavam excessivo amadurecimento aos três meses. Dessa maneira, o período de armazenamento foi estabelecido em função da evolução do amadurecimento conforme o comportamento da cultivar e da condição de arma- 
zenamento. Foram realizadas duas avaliações em momentos distintos para todos os tratamentos, sendo uma na abertura das minicâmaras e a outra após cinco dias de exposição dos frutos à temperatura de $20^{\circ} \mathrm{C}$, com o objetivo de simular o processo de beneficiamento, transporte e comercialização.

A firmeza de polpa, sólidos solúveis totais (SST) e a acidez titulável foram determinados como descritos em BRACKMANN et al. (1999). Frutos com manchas de diâmetro maior que $0,5 \mathrm{~cm}$ e com característica típica de ataque de microrganismos foram considerados podres. A avaliação de distúrbios fisiológicos internos dos frutos foi realizado através de diversos cortes transversais, expondo totalmente a polpa para observação visual. A avaliação organoléptica foi realizada procurando detectar a presença de sabor e aroma estranhos nos frutos por um painel de quatro pessoas, previamente treinadas. As médias dos tratamentos foram comparados pelo teste de Duncan com 5\% de probabilidade de erro, sendo que cada cultivar foi analisada separadamente como um experimento independente. Os valores expressos em porcentagem foram transformados pela fórmula arc.sen $\sqrt{x / 100}$ antes da análise estatística.

\section{RESULTADOS E DISCUSSÃO}

Em AC 2/7 ( $\mathrm{kPa}$ de $\left.\mathrm{O}_{2} / \mathrm{CO}_{2}\right)$, a firmeza de polpa e a acidez titulável (Tabela 1 e 2) dos frutos na abertura das minicâmaras mantiveram-se mais elevadas na temperatura de $-0,5^{\circ} \mathrm{C}$ em relação à temperatura de $0,5^{\circ} \mathrm{C}$, demonstrando ser a temperatura um fator de grande importância no controle do amadure- cimento do kiwi. A redução da temperatura diminui a atividade respiratória dos frutos (BEHRSING $\boldsymbol{e t}$ al., 1996) reduzindo a perda de firmeza (MERINO \& URIARTE, 1989) e acidez titulável (BURTON, 1982) durante o armazenamento.

As condições de AC, na abertura da câmara, mantiveram a firmeza de polpa, para ambas as cultivares, em valores mais elevados do que o armazenamento refrigerado (Tabela 1 e 2). Tais resultados já são bastante conhecidos, e evidenciam o efeito benéfico do baixo $\mathrm{O}_{2}$ e alto $\mathrm{CO}_{2}$ na redução do metabolismo respiratório dos frutos (MITCHELL $\boldsymbol{e t}$ al., 1982; McDONALD \& HARMAN, 1982; WATKINS \& ZHANG, 1996; SHIINA et al., 1997). Concentrações de $2 \mathrm{kPa}$ de $\mathrm{O}_{2}$ e $7 \mathrm{kPa}$ de $\mathrm{CO}_{2}$ mantiveram a firmeza de polpa mais elevada que $2 \mathrm{kPa}$ de $\mathrm{O}_{2}$ e $5 \mathrm{kPa}$ de $\mathrm{CO}_{2}$, quando o etileno foi absorvido, nas duas cultivares e em todas as avaliações.

O processo de eliminação de etileno com filtros químicos demonstrou grande eficiência na manutenção da firmeza e acidez dos frutos em condições de AC (Tabela 1 e 2), apresentando acentuado efeito quando utilizado na condição de $2 \mathrm{kPa}$ de $\mathrm{O}_{2}$ e $7 \mathrm{kPa}$ de $\mathrm{CO}_{2}$. Nessa condição, o período de armazenamento pode ser de, no mínimo, oito meses, pois foi observado que após oito meses e mais cinco dias a $20^{\circ} \mathrm{C}$, os frutos permaneciam com firmeza de polpa muito acima de $5 \mathrm{~N}$, considerada ideal para o consumo, conforme LEGARRAGA (1996). Resultados evidenciando o efeito benéfico do processo de absorção de etileno foram observados por vários autores (TONINI et al., 1989; MITCHELL, 1990; BONGHI et al., 1996; REDGWELL, 1996).

Tabela 1 - Firmeza de polpa, acidez titulável, sólidos solúveis totais e podridões de kiwi, cv. Bruno, após três meses de armazenamento refrigerado e mais cinco dias a $20^{\circ} \mathrm{C}$. Santa Maria, RS, 1997.

\begin{tabular}{|c|c|c|c|c|c|c|c|c|c|c|}
\hline \multicolumn{3}{|c|}{ TRATAMENTOS } & \multicolumn{2}{|c|}{$\begin{array}{l}\text { Firmez de Polpa } \\
\text { (N) }\end{array}$} & \multicolumn{2}{|c|}{$\begin{array}{l}\text { Acidez Titulável } \\
\text { (Cmol/L) }\end{array}$} & \multicolumn{2}{|c|}{$\begin{array}{l}\text { SST } \\
\left({ }^{\circ} \mathrm{B}\right)\end{array}$} & \multicolumn{2}{|c|}{$\begin{array}{l}\text { Podridão } \\
(\%)\end{array}$} \\
\hline Temp. & $\mathrm{kPaP}_{2} / \mathrm{kPaCO}_{2}$ & Etileno & $\begin{array}{l}\text { Saída da } \\
\text { câmara }\end{array}$ & $\begin{array}{l}+5 \text { dias } \\
\text { a } 20^{\circ} \mathrm{C}\end{array}$ & $\begin{array}{l}\text { Saída da } \\
\text { câmara }\end{array}$ & $\begin{array}{l}+5 \text { dias } \\
\text { a } 20^{\circ} \mathrm{C}\end{array}$ & $\begin{array}{l}\text { Saída da } \\
\text { câmara }\end{array}$ & $\begin{array}{l}+5 \text { dias } \\
\text { a } 20^{\circ} \mathrm{C}\end{array}$ & $\begin{array}{l}\text { Saída da } \\
\text { câmara }\end{array}$ & $\begin{array}{l}+5 \text { dias } \\
\text { a } 20^{\circ} \mathrm{C}\end{array}$ \\
\hline$-0,5$ & $2 / 5$ & Com absorção & $32,90 \mathrm{~b} *$ & $12,78 \mathrm{~b}$ & $27,18 \mathrm{cb}^{*}$ & $25,85 \mathrm{bc}$ & $13,00 \mathrm{cb}^{*}$ & $14,16 \mathrm{cb}$ & 0 & $0 \mathrm{~d}^{*}$ \\
\hline$-0,5$ & $2 / 5$ & Sem absorção & $12,15 \mathrm{c}$ & $4,75 \mathrm{~cd}$ & $25,93 \mathrm{fed}$ & $22,46 \mathrm{de}$ & $12,90 \mathrm{~cd}$ & $13,96 \mathrm{~cd}$ & 0 & $0 \mathrm{~d}$ \\
\hline$-0,5$ & $2 / 7$ & Com absorção & $43,02 \mathrm{a}$ & $22,58 \mathrm{a}$ & $27,66 \mathrm{~b}$ & $25,23 \mathrm{bc}$ & $12,46 \mathrm{e}$ & $13,70 \mathrm{e}$ & 0 & $0 \mathrm{~d}$ \\
\hline$-0,5$ & $2 / 7$ & Sem absorção & $15,53 \mathrm{c}$ & $7,20 \mathrm{c}$ & $26,43 \mathrm{~cd}$ & $21,46 \mathrm{e}$ & $12,60 \mathrm{ed}$ & $13,66 \mathrm{e}$ & 0 & $0 \mathrm{~d}$ \\
\hline$-0,5$ & $\mathrm{AR}$ & Com absorção & $10,67 \mathrm{~d}$ & $7,11 \mathrm{c}$ & $29,88 \mathrm{a}$ & $27,15 \mathrm{a}$ & $13,83 \mathrm{a}$ & $14,26 \mathrm{~b}$ & 0 & $2,22 \mathrm{c}$ \\
\hline$-0,5$ & $\mathrm{AR}$ & Sem absorção & $9,05 \mathrm{de}$ & $5,94 \mathrm{c}$ & $25,84 \mathrm{fed}$ & $26,21 \mathrm{ab}$ & $14,00 \mathrm{a}$ & $14,73 \mathrm{a}$ & 0 & $4,44 \mathrm{bc}$ \\
\hline$-0,5$ & $\mathrm{AR}$ & Com Ventilação & 9,96 de & $6,06 \mathrm{c}$ & 26,16 ed & $24,94 \mathrm{bc}$ & $13,76 \mathrm{a}$ & 13,86 ed & 0 & $2,22 \mathrm{c}$ \\
\hline$+0,5$ & $2 / 5$ & Sem absorção & $6,87 \mathrm{e}$ & $2,29 \mathrm{~d}$ & $25,43 \mathrm{fe}$ & $22,11 \mathrm{de}$ & $13,33 \mathrm{~b}$ & $13,96 \mathrm{~cd}$ & 0 & $11,08 \mathrm{a}$ \\
\hline$+0,5$ & $2 / 7$ & Sem absorção & $11,13 \mathrm{~d}$ & $4,58 \mathrm{~cd}$ & $25,11 \mathrm{f}$ & $23,17 \mathrm{~d}$ & $12,93 \mathrm{~cd}$ & $14,03 \mathrm{~cd}$ & 0 & $6,66 \mathrm{ab}$ \\
\hline \multicolumn{3}{|c|}{ Coeficientes de Variação (\%) } & 11,79 & 20,73 & 1,83 & 2,71 & 1,56 & 0,87 & - & 45,73 \\
\hline
\end{tabular}

*Médias não seguidas pela mesma letra na vertical diferem pelo teste de Duncan em nível de 5\% de probalidade do erro. 
Tabela 2 - Firmeza de polpa e acidez titulável de kiwi, cv. Hayward, após três e oito meses de armazenamento refrigerado e mais cinco dias a $20^{\circ} \mathrm{C}$. Santa Maria, RS, 1997.

\begin{tabular}{|c|c|c|c|c|c|c|c|c|c|c|}
\hline \multicolumn{3}{|c|}{ TRATAMENTOS } & \multicolumn{4}{|c|}{$\begin{array}{l}\text { FIRMEZA DE POLPA } \\
(\mathrm{N})\end{array}$} & \multicolumn{4}{|c|}{$\begin{array}{l}\text { ACIDEZ TITULÁVEL } \\
(\mathrm{Cmol} / \mathrm{L})\end{array}$} \\
\hline & & & \multicolumn{2}{|c|}{3 meses } & \multicolumn{2}{|c|}{8 meses } & \multicolumn{2}{|c|}{3 meses } & \multicolumn{2}{|c|}{8 meses } \\
\hline Temp. & $\mathrm{AP}_{2} / \mathrm{kPaCO}_{2}$ & Etileno & $\begin{array}{l}\text { Abertura } \\
\text { da câmara }\end{array}$ & $\begin{array}{l}+5 \text { dias } \\
\text { a } 20^{\circ} \mathrm{C}\end{array}$ & $\begin{array}{l}\text { Abertura } \\
\text { da câmara }\end{array}$ & $\begin{array}{l}+5 \text { dias } \\
\text { a } 20^{\circ} \mathrm{C}\end{array}$ & $\begin{array}{l}\text { Abertura } \\
\text { da câmara }\end{array}$ & $\begin{array}{l}+5 \text { dias } \\
\text { a } 20^{\circ} \mathrm{C}\end{array}$ & $\begin{array}{l}\text { Abertura } \\
\text { da câmara }\end{array}$ & $\begin{array}{l}+5 \text { dias } \\
\text { a } 20^{\circ} \mathrm{C}\end{array}$ \\
\hline$-0,5$ & $2 / 5$ & Com absorção & $52,22 b^{*}$ & $26,49 \mathrm{~b}$ & $18,43 \mathrm{~b}$ & $7,66 \mathrm{~b}$ & $22,49 b^{*}$ & $21,64 \mathrm{a}$ & 20,46 a & $18,23 \mathrm{a}$ \\
\hline$-0,5$ & $2 / 5$ & Sem absorção & $25,35 \mathrm{~d}$ & $10,20 \mathrm{c}$ & $4,96 \mathrm{~d}$ & $2,75 \mathrm{de}$ & $21,16 \mathrm{~cd}$ & $18,80 \mathrm{~cd}$ & $17,34 \mathrm{~b}$ & $16,13 \mathrm{~b}$ \\
\hline$-0,5$ & $2 / 7$ & Com absorção & $59,53 \mathrm{a}$ & $38,92 \mathrm{a}$ & $40,47 \mathrm{a}$ & $9,54 \mathrm{a}$ & 23,16 a & $21,88 \mathrm{a}$ & 19,86 a & 17,83 a \\
\hline$-0,5$ & $2 / 7$ & Sem absorção & $33,07 \mathrm{c}$ & $12,33 \mathrm{c}$ & $7,94 \mathrm{c}$ & $4,53 \mathrm{c}$ & $21,43 \mathrm{c}$ & $19,34 \mathrm{cb}$ & $16,71 \mathrm{~b}$ & $16,23 \mathrm{~b}$ \\
\hline$-0,5$ & $\mathrm{AR}$ & Com absorção & $18,05 \mathrm{e}$ & $9,54 \mathrm{c}$ & - & - & $22,45 \mathrm{~b}$ & $21,23 \mathrm{a}$ & - & - \\
\hline$-0,5$ & $\mathrm{AR}$ & Sem absorção & $15,27 \mathrm{e}$ & $9,44 \mathrm{c}$ & - & - & $21,32 \mathrm{c}$ & $19,64 \mathrm{~b}$ & - & - \\
\hline$-0,5$ & $\mathrm{AR}$ & Com Ventilação & $17,83 \mathrm{e}$ & $9,56 \mathrm{c}$ & - & - & $21,72 \mathrm{c}$ & $19,82 \mathrm{~b}$ & - & - \\
\hline$+0,5$ & $2 / 5$ & Sem absorção & $28,40 \mathrm{~cd}$ & $10,76 \mathrm{c}$ & $4,07 \mathrm{~d}$ & $2,37 \mathrm{e}$ & $20,53 \mathrm{de}$ & $18,33 \mathrm{~d}$ & $17,24 \mathrm{~b}$ & $15,45 \mathrm{c}$ \\
\hline$+0,5$ & $2 / 7$ & Sem absorção & $25,59 \mathrm{~d}$ & $12,39 \mathrm{c}$ & $4,34 \mathrm{~d}$ & $3,91 \mathrm{~cd}$ & $20,22 \mathrm{e}$ & $18,80 \mathrm{~cd}$ & $16,91 \mathrm{~b}$ & $15,98 \mathrm{cb}$ \\
\hline \multicolumn{3}{|c|}{ Coeficientes de Variação (\%) } & 10,98 & 10,71 & 10,96 & 14,12 & 1,78 & 1,92 & 2,38 & 1,96 \\
\hline
\end{tabular}

*Médias não seguidas pela mesma letra na vertical diferem pelo teste de Duncan em nível de 5\% de probalidade do erro.

$\mathrm{O}$ efeito conjunto da baixa temperatura, baixo $\mathrm{O}_{2}$, alto $\mathrm{CO}_{2}$ e absorção de etileno demonstrou grande eficiência na retenção da firmeza de polpa e acidez titulável dos frutos, demonstrando que a perda de firmeza e acidez durante o armazenamento está diretamente relacionada com a atividade respiratória dos frutos, sendo a respiração reduzida com baixas temperaturas (BEHRSING et al., 1996), baixas concentrações de $\mathrm{O}_{2}$ (SHIINA et al., 1997), altas de $\mathrm{CO}_{2}$ (WATKINS \& ZHANG, 1996) e absorção de etileno (SOLOMOS \& BIALE, 1975).

Nas condições de armazenamento refrigerado, o período de armazenamento não pode ser superior a três meses para as duas cultivares, pois os frutos mostraram-se com elevada perda de firmeza. Conforme WARRINGTON \& WESTON (1990), as condições de armazenamento refrigerado não são indicadas para o armazenamento por longos períodos, devido especialmente à elevada perda de firmeza e incidência de podridões, o que compromete a qualidade dos frutos. Sob refrigeração, os processos de eliminação de etileno com filtros químicos e ventilação da câmara frigorífica não mostraram eficiência na retenção da perda da firmeza dos frutos, em comparação com o armazenamento refrigerado sem eliminação de etileno. Isso demonstra que, nas condições de AR, o metabolismo é intenso, e mesmo com a eliminação de etileno, as condições favorecem a rápida perda de firmeza.

Em todas as condições de armazenamento, ocorreu uma evolução do teor dos açúcares (Tabelas 1 e 3), que atingiu níveis superiores a $12^{\circ} \mathrm{B}$, demonstrando que o ponto de maturação na colheita e as condições de armazenamento proporcionaram valores de SST satisfatórios para um bom sabor. As condições de AC, de modo geral, não apresentaram efeito consistente sobre os níveis de SST, confirmando resultados obtidos por MITCHELL et al., (1983), ficando abaixo dos valores observados para AR evidenciando um menor uso de reservas.

Nas avaliações realizadas aos três meses, na abertura das câmaras, não ocorreu incidência de podridões (Tabela 1 e 3). Após a exposição dos frutos a $20^{\circ} \mathrm{C}$, porém, houve desenvolvimento de podridões nos frutos mantidos em $\mathrm{AR}$ e em $\mathrm{AC}$ a $0,5^{\circ} \mathrm{C}$. Nos frutos armazenados em $\mathrm{AC}$ a $-0,5^{\circ} \mathrm{C}$, mesmo após a exposição por cinco dias à temperatura ambiente, não houve desenvolvimento de podridões para a cultivar Bruno. Condições de AC em baixa temperatura retardam o amadurecimento e senescência, reduzindo a susceptibilidade dos frutos aos microrganismos deterioradores. Observa-se, ainda, que para a cv. Bruno (Tabela 1) a redução da temperatura é mais determinante que a utilização da AC no controle de podridões. Possivelmente, essa maior incidência de podridões na temperatura de $0,5^{\circ} \mathrm{C}$ deve-se à avançada maturação dos frutos, predispondo-os ao ataque de fungos deterioradores; além disso, a baixa temperatura inibe o desenvolvimento dos microrganismos, apresentando por isso eficiência na redução de podridões pós-colheita de kiwi (SAWADA et al., 1993).

Durante o armazenamento refrigerado, os processos de eliminação de etileno com filtros químicos e ventilação da câmara apresentaram redução da incidência de podridões, em relação ao armaze- 
Tabela 3 - Sólidos solúveis totais e podridões de kiwi, cv. Hayward, após três e oito meses de armazenamento refrigerado e mais cinco dias a $20^{\circ} \mathrm{C}$. Santa Maria, RS, 1997.

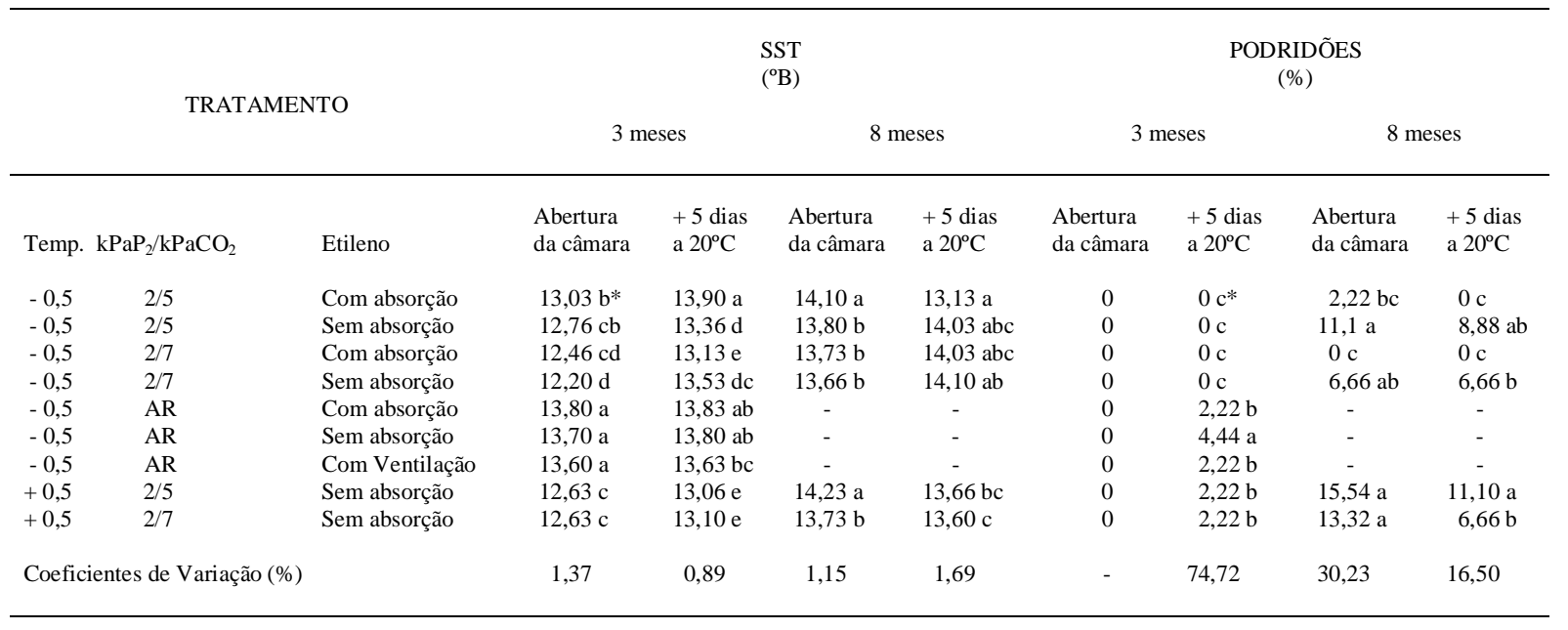

*Médias não seguidas pela mesma letra na vertical diferem pelo teste de Duncan em nível de $5 \%$ de probalidade do erro.

namento refrigerado sem absorção (Tabela 1 e 3 ). $\mathrm{Na}$ avaliação realizada para a cv. Hayward, aos oito meses (Tabela 3), a incidência de podridões foi reduzida na temperatura de $-0,5^{\circ} \mathrm{C}$, sendo inibida com a absorção de etileno. A utilização de absorvedores em AC mostrou ser de fundamental importância para redução da incidência de podridões. Segundo SOMMER et al. (1983), o etileno induz os frutos ao amadurecimento e predispõe-os ao ataque de microrganismos. Isso se deve à degradação das pectinas, que diminuem a resistência da parede celular ao ataque de fungos.

Foram identificados os fungos Botrytis cinerea, Penicillium expansum e Sclerotinia sclerotiorum, com predominância do primeiro. Esses resultados confirmam os encontrados por diversos autores (BROOK, 1992; WARD et al., 1996; POOLE et al., 1996), que caracterizaram Botrytis cinerea como o principal agente causal de podridões em pós-colheita de kiwi.

Nas avaliações realizadas, não foram verificados distúrbios fisiológicos, nem mesmo sabores e aromas estranhos nos frutos, demonstrando que as condições avaliadas estão dentro da faixa tolerável, pois não alteraram o metabolismo normal dos frutos, a integridade estrutural das células e não propiciaram o acúmulo de substâncias indesejáveis, responsáveis por sabor e aromas anormais. Concentrações de $2 \mathrm{kpa}$ de $\mathrm{O}_{2}$ e $7 \mathrm{kpa}$ de $\mathrm{CO}_{2}$ não foram suficientes para causar danos por alto $\mathrm{CO}_{2}$, conforme descrito por HARMAN \& McDONALD (1983), e por baixo $\mathrm{O}_{2}$ (KADER, 1997). Não foi observado também o sinergismo entre alto $\mathrm{CO}_{2}$ e presença de etileno, na manifestação de distúrbios fisiológicos, conforme observado por ARPAIA et al. (1985).

\section{CONCLUSÕES}

A temperatura de $-0,5^{\circ} \mathrm{C}$ mantém superior qualidade dos kiwis comparada a $0,5^{\circ} \mathrm{C}$;

$\mathrm{O}$ armazenamento em AC, comparado ao $\mathrm{AR}$, retarda a maturação e prolonga o período de conservação, sendo $2 \mathrm{kPa}$ de $\mathrm{O}_{2} / 7 \mathrm{kPa}$ de $\mathrm{CO}_{2}$ a melhor condição de AC;

A absorção química de etileno mantém melhor qualidade dos frutos, no entanto, a eliminação do etileno por ventilação da câmara não apresenta efeito.

\section{REFERÊNCIAS BIBLIOGRÁFICAS}

ARPAIA, M.L., MITCHELL, F.G., KADER, A.A., et al. Effects of $2 \% \mathrm{O}_{2}$ and varying concentrations of $\mathrm{CO}_{2}$ with or without $\mathrm{C}_{2} \mathrm{H}_{4}$ on the storage performance of kiwifruit. J Amer Soc Hort Sci, Alexandria, USA, v.110, n.2, p.200-203, 1985.

BEHRSING, J.P., TOMKINS, R.B., HUTCHIN, J.M., et al. Effect of temperature and size reduction on respiratory activity and shelf-life of vegetables. In: INTERNATIONAL POSTHARVEST SCINCE CONFERENCE, 1996, Taupo. Abstracts... Taupo : ISHS, 1996. 544p. p.500.

BONGHI, C., PAGNI, S., VIDRIH, R., et al. Cell wall hydrolases and amylase in kiwifruit softening. Postharvest Biology and Technology, Amsterdam, v.9, n.1, p.19-29, 1996.

BRACKMANN, A., MAZARO, S.M., PETRY, C. Qualidade de kiwi sob condições de armazenamento refrigerada e atmosfera controlada. Revista Brasileira de Fruticultura, Jaboticabal, v.21, n.1, p.45-48, 1999.

BROOK, P.J. Botrytis stem-end rot and other storage diseases of kiwifruit. Acta Horticulturae, Wageningen, v.297, n.2, p.545-550, 1992.

Ciência Rural, v. 30, n. 6, 2000. 
BURTON, W.G. Postharvest physiology of food crops. New York: Longman, 1982. 339p.

EMPRESA DE PESQUISA AGROPECUÁRIA E EXTENSÃO RURAL DE SANTA CATARINA. Normas técnicas para cultivo do quivi no sul do Brasil. Florianópolis : EPAGRI, 1996. 38p. (EPAGRI, Sistema de produção, 25).

HARMAN, J.E., McDONALD, B. Controlled atmosphere storage of kiwifruit: Effects on storage life and fruit quality. Acta Horti culturae, Wageningen, v.138, p.195-201, 1983.

HARRIS, S. The refrigerated export chain of kiwifruit (Actinidia deliciosa) from New Zealand. Bull Inst Int Froid, Annexe, v.1, p.157-164, 1976

HARRIS, S., REID, M.S. Techniques for improving the storage life of kiwifruit (Actinidia chinensis). Auckland: Auckland Industrial Development Division, DSIR, 1981. 23p. (Publication G120).

KADER, A.A. Summary of requirements and recommendation for fruits other than apples and pears. In: INTERN CONTROLLED ATMOSPH. RES. CONF. 7, 1997, Davis, California. Proceedings... Davis : University of California, 1997. v.3. 263p. p.01-34

LEGARRAGA D.M. Cosecha, conservación y normas de embalajede kiwis. In: SIMPÓSIO BRASILEIRO DA CULTURA DO KIWI, 1, 1994, Farroupilha, RS. Anais... Bento Gonçalves : EMBRAPA-CNPUV, 1996. 48p. p.25-

McDONALD, B., HARMAN, J.E. Controlled atmosphere storage of kifruit. In: Effect on fruit firmness and storage life. Scientia Hort, Amsterdam, v.17, p.113-123, 1982.

MERINO, D.M., URIARTE, C. Conservación del kiwi Fruticultura Profesional, Barcelona, n.22, p.35-42, 1989.

MITCHELL, F.G. Postharvest physiology and technology of kiwi. Acta Horticulturae, Wageningen, n.282, p.291-307, 1990.

MITCHELL, F.G., ARPAIA, M.L., MAYER, G. Modifiedatmosphere storage of kiwifruit (Actinidia chinensis). In: CONTROLLED ATMOSPHERE RES. CONF., 3, 1982, Beaverton. Proceedings... Beaverton, 1982. v.1. p.235238. (Symposium Series).

MITCHELL, F.G., ARPAIA, M.L., MAYER, G. Maturity measurement of "Hayward" kiwifruit (Actinidia chinenses Planch.). HortScience, Alexandria, v.18, n.4, p.615, 1983.

PARMENTIER, V.M., PROFT. M.M.P. Condition of kiwifruit on the European market after storage under $\mathrm{CA}$ in New Zealand. In: INTERN. CONTROLLED ATMOSPH. RES. CONF, 7, 1997, Davis, California. Proceedings... Davis : University of California, 1997. v.3. 263p .p.62-68.
POOLE, P.R., McLEOD, L.C., WHITMORE, et al. Preharvest control of Botrytis cinerea rots in stored kiwifruit. In INTERNATIONAL POSTHARVEST SCINCE CONFERENCE, 1996, Taupo. Abstracts... Taupo : ISHS, 1996. 544p. p.71-76.

REDGWELL, R.J. Cell wall synthesis in kiwifruit following postharvest ethylene treatment. Phytochemistry, Oxford, v.41, n.2, p.407-413, 1996.

SAWADA, T. SEO, Y., MORISHIMA, H. Studies on storage and ripening of kiwifruit. Journal of the Japanese Society of Agricultural Machinery, v.55, n.2, p.59-68, 1993.

SHIINA, T., YAMAUCHI, H. , HARUENKIT, Effects of temperature and gas concentration on the respiration of fruits and vegetables. In: INTERN. CONTROLLED ATMOSPH. RES. CONF, 7, 1997, Davis, California. Proceedings... Davis : University of California, 1997. v.1. n.1. 159p. p.7176.

SOLOMOS, T. BIALE, J.B. Respiration and fruit ripening Facteurs et regulation the la maturation des fruits. In: COLLOQUES INTERNATIONAUX DU CENTRE NATIONAL DE LA RECHERCHE SCIENTIFIQUE, 1974 Paris. Proceedings... Paris : CNRS, 1975. n.238. 369p. p.221-228.

SOMMER, N.F., FORTLAGE, R.J., EDWARDS, D.C Minimizing postharvest diseases of kiwifruit. California Agriculturae, v.37, n.1, p.16-18, 1983.

TONINI, G., BRIGATI, S., CACCIONI, D. CA Storage of kiwifruit: influence on rots and storability. In INTERNATIONAL CONTROLLED ATMOSPHERE RESEARCH CONFERENCE, 5, 1989, Wenatchee, Washington. Proceedings... Moscow : University of Idaho, 1989. v.2. 374p. p.69-76.

TONINI, G., BASSI, F., PRONI, R. CA storage of kiwifruit at different $\mathrm{O}_{2}$ and $\mathrm{CO}_{2}$ levels: Influence on Botrytis cinerea Phialophora spp. Rots and fruit maturity. In: INTERNATIONAL POSTHARVEST SCIENCE CONFERENCE, 1996, Taupo. Abstracts... Taupo : ISHS, 1996. 544p. p. 211

WARD, B.G., HILL, R.A., POOLE, P.R. Postharvest application of natural products to control Botrytis storage rot in kiwifruit. In: INTERNATIONAL POSTHARVEST SCINCE CONFERENCE, 1996, Taupo. Abstracts... Taupo : ISHS, 1996. 544 p. p. 97.

WARRINGTON, I.J., WESTON, G.C. Kiwifruit science and management. Auckland : Ray Richards, 1990. 576p.

WATKINS, C.B., ZHANG, J. Metabolic Responses of fruit to carbon dioxide. In: INTERNATIONAL POSTHARVEST SCINCE CONFERENCE, 1996, Taupo. Abstracts... Taupo: ISHS, 1996. 544p. p.345-350. 